\title{
Proximity-induced magnetism in Pt layered with rare-earth-transition-metal ferrimagnetic alloys
}

\author{
C. Swindells $\odot,{ }^{1}$ B. Nicholson, ${ }^{1}$ O. Inyang $\odot,{ }^{1}$ Y. Choi $\odot,{ }^{2}$ T. Hase $\odot,{ }^{3}$ and D. Atkinson $\oplus^{1, *}$ \\ ${ }^{1}$ Department of Physics, Durham University, South Road, Durham DH1 3LE, United Kingdom \\ ${ }^{2}$ Advanced Photon Source, Argonne National Laboratory, Argonne, Illinois 60439, USA \\ ${ }^{3}$ Department of Physics, University of Warwick, Coventry CV4 7AL, United Kingdom
}

(Received 12 June 2020; revised 21 July 2020; accepted 29 July 2020; published 20 August 2020)

\begin{abstract}
The proximity-induced moment (PIM) in heavy metal layers may play a significant role in heterostructured spintronic systems. In particular, the PIM of a heavy metal adjacent to a magnetic layer has been linked to interfacial spin transport behavior. Element-resolved $\mathrm{x}$-ray magnetic measurements were used to investigate PIM in Pt layered with two different rare-earth (RE):3d transition-metal (TM) ferrimagnetic alloys in which the net moment was dominated by either the RE or the TM at room temperature. We observed significant PIM in Pt confined to a 2-nm interfacial region for $\mathrm{Pt} / \mathrm{Co}_{77} \mathrm{Gd}_{23}$ and $\mathrm{Pt} /\left(\mathrm{Fe}_{50} \mathrm{Co}_{50}\right)_{77} \mathrm{Gd}_{23}$ and, in both cases, the PIM was parallel to the TM sublattice rather than the RE or the net moment. Our results highlight the prominence of the $d-d$ mediated interactions between the Pt and the constituents of the ferrimagnetic RE:TM alloys over the net macroscopic moment.
\end{abstract}

DOI: 10.1103/PhysRevResearch.2.033280

In the decades since the discovery of giant magnetoresistance, a range of effects have emerged in ferromagnetic/ nonmagnetic systems (FM/NM) that open up new avenues for physical understanding and opportunities for applications in spintronics technology. Many of these phenomena are associated with the interfaces within such FM/NM systems. These include enhanced spin-orbit interactions (SOI), interfacial anisotropy, Dzyaloshinskii-Moriya interaction (DMI), and electronic hybridization that creates a proximity-induced moment (PIM) or magnetic proximity effect (MPE) in nonmagnetic heavy metals. PIM is known to enhance the magnetooptical response in $\mathrm{Co} / \mathrm{Pt}$ systems [1], and the relationship between PIM and other interfacial phenomena has been explored [2,3]. The propagation of spin current, both from a magnetic layer into a NM layer, that enhances magnetic damping and, via the spin Hall effect, from a heavy metal into a magnetic layer, that enables spin-orbit torques (SOT), is well established in ferromagnetic systems and has been demonstrated in ferrimagnetic (FiM) systems [4,5]. Such spin transport has also been shown to depend critically upon the details of the interface [6-11]. However, debate continues about the role of PIM in such spin transport physics, both with respect to metallic FM/NM systems $[12,13]$ and in insulating ferrimagnets layered with heavy metals (FiM/NM) [14-19]. Understanding the physical basis of PIM in the nonmagnetic heavy metals that play a key role in spintronics in relation to

\footnotetext{
*del.atkinson@durham.ac.uk

Published by the American Physical Society under the terms of the Creative Commons Attribution 4.0 International license. Further distribution of this work must maintain attribution to the author(s) and the published article's title, journal citation, and DOI.
}

ferro- and ferrimagnetic layers is, therefore, both significant and timely.

Experimental studies using element-specific x-ray magnetic circular dichroism (XMCD) have established the presence of a significant proximity-induced moment in nonmagnetic heavy metals layered with ferromagnetic transition metals [20-23]. In contrast, debate remains for NM heavy metals layered with insulating ferrimagnetic oxides. XMCD studies of Pt layered with several different ferrimagnetic oxides, including $\mathrm{Fe}_{3} \mathrm{O}_{4}$ and $\mathrm{CoFe}_{2} \mathrm{O}_{4}$, show no measurable magnetic polarization of the Pt layer [18,24,25], while for $\mathrm{Pt}$ in contact with yttrium iron garnet, XMCD shows an induced moment in $\mathrm{Pt}$, but there are conflicting reports about the magnitude of the moment induced in Pt [24,26]. More recently, the absence of PIM in Pt in contact with $\mathrm{CoFe}_{2} \mathrm{O}_{4}$ has been recently contradicted by indirect evidence from anisotropic magnetoresistance measurements and supported by density functional theory, indicating a small induced moment on Pt [19,27].

These differences in the PIM of Pt layered with either metallic ferromagnets or ionic ferrimagnets may be linked to the nature of the magnetic moments or the mechanism of exchange ordering. For the oxide ferrimagnets, the magnetic moments are localized to lattice sites and magnetic sublattices align antiparallel via superexchange, mediated locally by hybridization of individual orbitals. In contrast, in transitionmetal ferromagnets the moments are less localized and the $d-d$ exchange interactions, which give rise to parallel alignment, are mediated by the conduction electrons. Investigating PIM in alternative magnetic systems can, therefore, provide new insights into the role of the moments and the exchange interactions on the PIM in heavy metals resulting from hybridization at the interface.

Here, PIM in Pt was studied in a system layered with ferrimagnetic rare-earth/transition-metal (RE:TM) alloys, 
allowing an investigation of PIM in a heavy metal coupled to a system with metallic magnetism but with antiferromagnetic alignment of the transition and rare-earth-metal sublattices.

RE:TM ferrimagnetic thin-film systems have been extensively studied, most recently due to their potential in spintronic applications. With different temperature dependencies of the magnetic moments and antiparallel coupling between the TM and RE sublattices in multilayers [28] and alloys [29], compensation points occur with net zero magnetization and angular momentum [30] at a given temperature or composition [31]. These compensation points lead to useful phenomena, such as fast domain wall motion [32,33], thermally induced switching $[34,35]$, vanishing skyrmion Hall angles [36], and self-focusing skyrmion racetracks [37], while efficient spin-orbit torque switching around compensation has been observed in studies of RE:TM alloys with bulk perpendicular anisotropy (PMA) [38-42], the onset of which may be controlled by the RE content [43]. Recently, it has also been shown that domain walls and compensation temperatures can be altered with ion bombardment [44].

SOT switching of a magnetic structure requires the efficient generation and propagation of a pure spin current from a heavy metal layer into the magnetic material. Pt is most commonly used due to its large spin orbit coupling (SOC), which creates spin current via the spin Hall effect (SHE) with the flow of charge current $[45,46]$.

In contrast to ferromagnetic metals and oxide ferrimagnets, the presence or absence of PIM in Pt in contact with metallic RE:TM ferrimagnets is unknown. It is also not known whether any induced moment in Pt would be directly coupled to one of the ferrimagnetic sublattices or follows the net magnetization of the alloy. Experimental insights into the PIM of a heavy metal (HM) in contact with RE:TM ferrimagnets can, therefore, provide a fuller understanding of PIM that may inform the role of PIM in spin transport.

In this article, we present a study of PIM in Pt layered with two RE:TM ferrimagnetic alloys. The alloys had compensation points above and below room temperature, respectively, in order to determine the presence and the orientation of the PIM in relation to the direction of RE or the TM sublattice moments and to the net magnetization. A CoGd alloy was used in which the RE moment dominated, while a CoFeGd alloy was selected with a net moment that is dominated by the transition metal at room temperature. Using element-specific resonant $\mathrm{x}$-ray analysis, both the direction of the induced $\mathrm{Pt}$ moment, with respect to the ferrimagnetic sublattices, and the profile of the Pt PIM as a function of distance from the interface with the ferrimagnet were determined.

Samples were grown by magnetron sputtering, with the ferrimagnetic alloys produced by cosputtering from $\mathrm{TM}$ and $\mathrm{Gd}$ targets. Atomic compositions were determined using energydispersive x-ray spectroscopy (EDS). Trilayered structures of $\mathrm{Pt}(5 \mathrm{~nm}) /$ FiM alloy $(15 \mathrm{~nm}) / \mathrm{Pt}(5 \mathrm{~nm})$ were studied, where the FiM alloy was either $\mathrm{Co}_{77} \mathrm{Gd}_{23}$ or $\left(\mathrm{Fe}_{50} \mathrm{Co}_{50}\right)_{77} \mathrm{Gd}_{23}$. At room temperature, the magnetization of the CoGd alloy was dominated by $\mathrm{Gd}$, while for $\mathrm{CoFeGd}$ it was dominated by the TM, the FiM alloy layers were amorphous, and the magnetization was in-plane.

Magnetic hysteresis loops, from a vibrating-sample magnetometer, superconducting quantum interference device

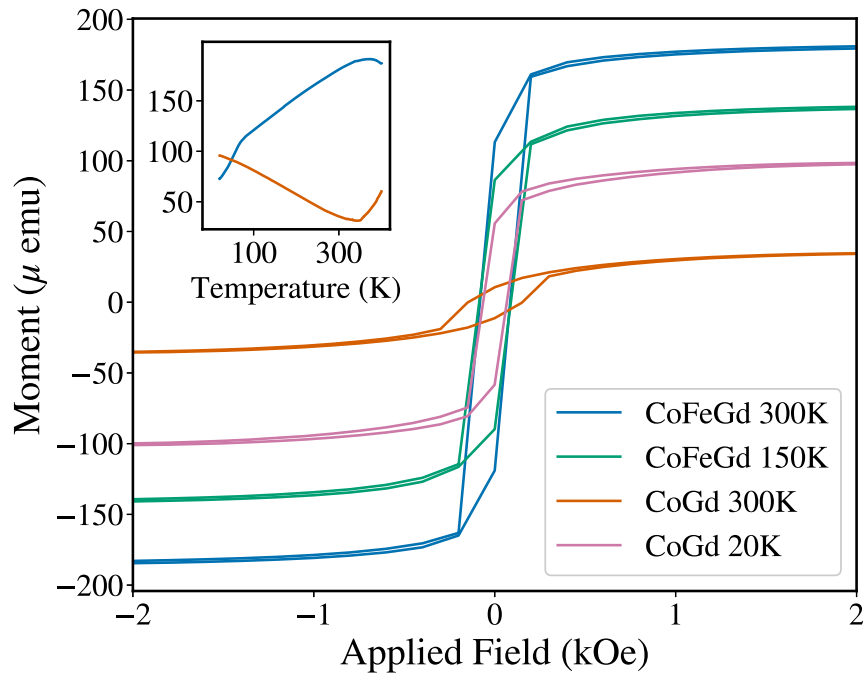

FIG. 1. Magnetic hysteresis for the two Pt/RE:TM/Pt ferrimagnetic alloys. The inset shows the saturation moment as a function of temperature for $\mathrm{Pt} / \mathrm{CoGd} / \mathrm{Pt}$ (orange) and $\mathrm{Pt} / \mathrm{CoFeGd} / \mathrm{Pt}$ (blue), which indicates compensation points above and below room temperature, respectively.

(VSM-SQUID) show the field dependence of the total magnetic moment at room temperature and low temperatures for each alloy, see Fig. 1, with the inset showing the temperature dependence of the net magnetization. The CoGd shows a minimum in the temperature dependence of the net moment that is characteristic of the magnetization compensation point, which in this case is above room temperature. It is also noted that a divergence in the coercive field was observed at the compensation point in similar alloys, explaining the enhanced coercivity of the CoGd alloy observed at $300 \mathrm{~K}$ [47]. At temperatures below compensation, the Gd moment dominates and the Gd sublattice will be aligned with the applied field, with the Co sublattice antiparallel. Above the compensation temperature the TM moment dominates the net moment, which is the case for the $\mathrm{CoFeGd}$ sample, which shows no compensation point down to $20 \mathrm{~K}$, the lowest temperature studied.

Resonant x-ray studies at the $\mathrm{Pt}$ and $\mathrm{Gd} L_{3}$ edges (11.56 and $7.24 \mathrm{keV}$, respectively) were undertaken on the 4-ID-D beamline of the Advanced Photon Source at Argonne National Laboratory. X-ray magnetic circular dichroism (XMCD) is a well-established technique to probe the magnetic moments of specific elements. Although determining values for moments using the sum rules for Gd is not accurately applicable [48], the sign of the dichroic spectra was used to determine the direction of the elemental magnetic moments with respect to the probe beam and applied field. Samples were also studied with grazing-incidence resonant reflectivity, with a magnetic field of $\pm 0.6 \mathrm{kOe}$ applied in-plane and along the beam axis, to obtain structural and magnetic information at the Pt $L_{3}$ edge. Analysis of the magnetic component of the resonant reflectivity allows a depth profile of the induced Pt magnetization to be determined. X-ray absorption spectroscopy (XAS) was measured using a fluorescence detector, with both left and right circularly polarized $\mathrm{x}$-ray intensities measured at each point. The measured XMCD signal was given by $\frac{I^{+}-I^{-}}{I^{+}+I^{-}}$, 

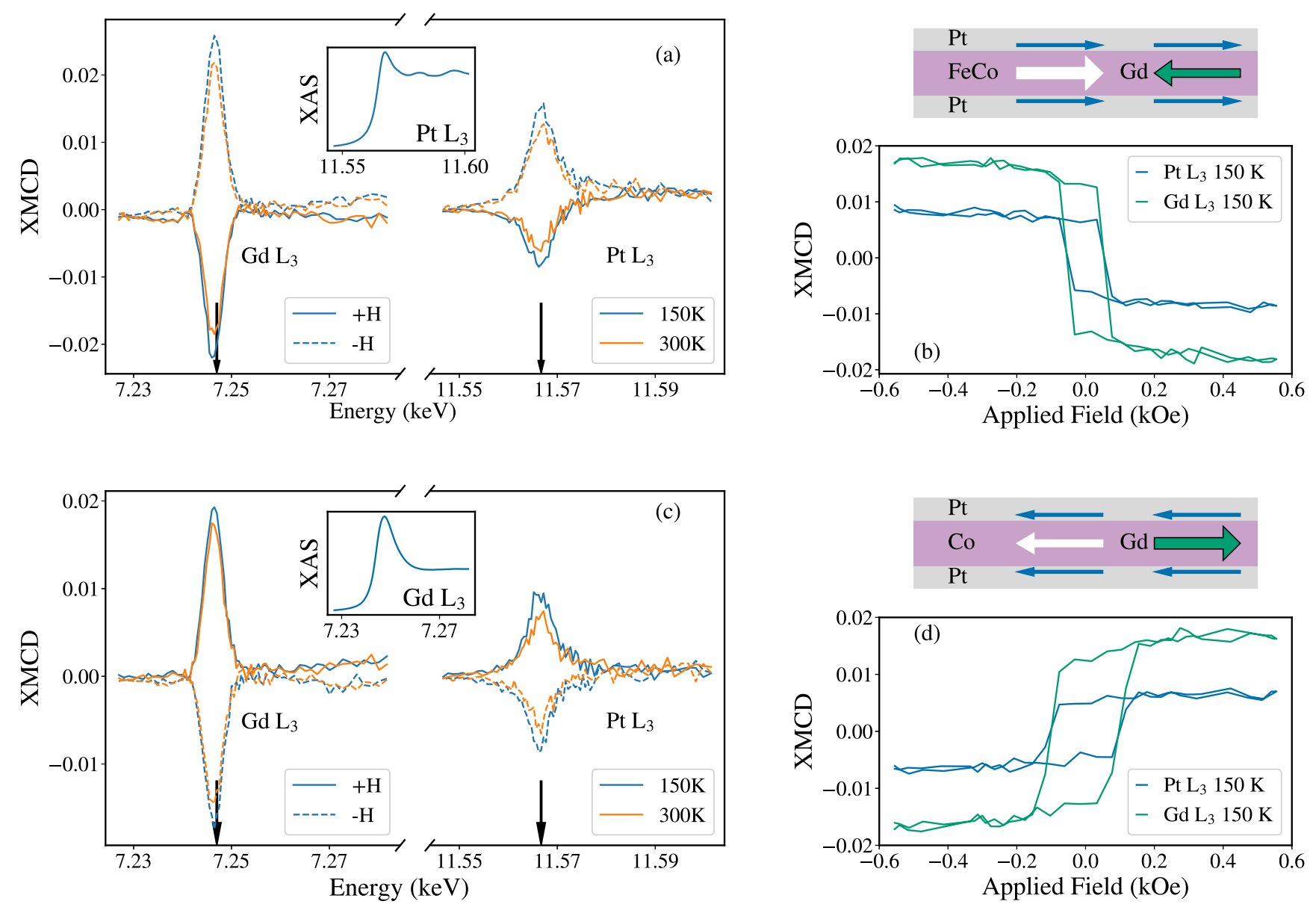

FIG. 2. Energy scans and element-specific hysteresis loops at the $L_{3}$ edges of $\mathrm{Pt}$ and $\mathrm{Gd}$ in a $\mathrm{Pt} / \mathrm{FiM}$ alloy/Pt structure with $\left(\mathrm{Fe}_{50} \mathrm{Co}_{50}\right)_{77} \mathrm{Gd}_{23}(\mathrm{a}, \mathrm{b})$ and $\mathrm{Co}_{77} \mathrm{Gd}_{23}(\mathrm{c}, \mathrm{d})$. Vertical arrows on the energy scans denote the energies at which the Gd/Pt specific hysteresis loops were taken. (Inset) X-ray absorption spectra over the $L_{3}$ edge of $\mathrm{Pt}$ (a) and $\mathrm{Gd}$ (c). Schematic illustrations show the determined orientation of the magnetic moments in the alloy.

where $I^{+}$and $I^{-}$denote the spectra with opposite circular polarizations, for a fixed magnetic field. The polarizationaveraged XAS is defined as $\left(I^{+}+I^{-}\right) / 2$.

Figure 2 presents the XMCD spectra, which show the maximum magnetic signals at the $\mathrm{Pt}$ and $\mathrm{Gd} L_{3}$ edges. These spectra probe the $p$-to- $d$ transitions and thus the Pt and Gd $5 d$ moments, respectively. Knowing the sign of the XMCD $L_{3}$ signal of the Gd sublattice and for the $\mathrm{Pt}$, and knowing which sublattice dominates the magnetization, the alignment of the PIM in the Pt with either the TM or RE sublattice was determined. This alignment is illustrated schematically in Fig. 2. Here, the x-ray energy range allowed the study of dichroic absorption at the $\mathrm{Gd} L_{3}$ edge. However, since other key works on XMCD of Gd have focused on the Gd $M_{5}$ edge, the relationship between the sign of the $M_{5}$ and $L_{3}$ dichroic edges and the direction of the magnetic moment needs to be briefly discussed. First, it was established in previous studies of ferromagnetic Gd that when the magnetization was aligned with the field, the sign of the dichroic spectrum at the $M_{5}$ edge was negative and at the $L_{3}$ edge was positive [48-50]. Therefore, for the $\mathrm{Gd} L_{3}$ edge, the dichroic spectrum is positive when the Gd magnetic moment is positively aligned with the magnetic field, and the spectrum is negative when the Gd moment is antiparallel to the applied magnetic field. Second, in ferrimagnetic CoGd alloys, where Co dominates the magnetization, the sign of the XMCD spectrum of the Gd $M_{5}$ edge was positive while the Co $L_{3}$ edge was negative [51], and across the compensation temperature, when the $\mathrm{Gd}$ moment dominates the magnetization, both the $\mathrm{Co}$ and $\mathrm{Gd}$ XMCD signals were reversed, i.e., the Co $L_{3}$ edge becomes positive and the Gd $M_{5}$ edge negative [29]. Bringing these facts together, for the antiparallel ferrimagnetic coupling of Co and Gd, the sign of both the Co $L_{3}$ edge XMCD spectrum and the $\mathrm{Gd} L_{3}$ edge spectrum will be the same, even though the magnetic moments of the two sublattices are antiparallel. Separately, it has also been shown that the sign of the $\mathrm{Pt}$ $\mathrm{XMCD} L_{3}$ edge in the Co/Pt system is the same as the Co $L_{3}$ edge, i.e., is negative for positive orientation of the Co magnetization [12,52]. In summary, when Gd is aligned parallel with the saturating field the Gd XMCD $L_{3}$ spectrum will be positive. In the case where $\mathrm{Pt}$ and $\mathrm{Gd}$ are aligned antiparallel, the $L_{3}$ XMCD spectra of both Gd and Pt will have the same sign. However, the alignment with respect to the magnetic field will depend on whether the Gd or the TM sublattice 
TABLE I. A summary of the relations between the signs of dichroic spectra, and the alignment of moments.

\begin{tabular}{lccc}
\hline \hline Element edges & Dichroic signs & Moments & Refs. \\
\hline Gd $M_{5}-\mathrm{Gd} L_{3}$ & Opposite & Parallel & {$[48-50]$} \\
$\operatorname{Gd} M_{5}-\mathrm{Co} L_{3}$ & Opposite & Antiparallel & {$[29,51]$} \\
$\operatorname{Co} L_{3}-\mathrm{Pt} L_{3}$ & Same & Parallel & {$[12,52]$} \\
$\operatorname{Gd} L_{3}-\mathrm{Co} L_{3}$ & Same & Antiparallel & \\
$\operatorname{Gd} L_{3}-\mathrm{Pt} L_{3}$ & Same & Antiparallel & \\
\hline \hline
\end{tabular}

dominates the magnetization. A simplified summary of these alignments is presented in Table I. Separately, to confirm this analysis, the sign of the Pt $L_{3}$ signal was compared with a reference $\mathrm{Co} / \mathrm{Pt}$ multilayer film in which the parallel alignment of the $\mathrm{Pt}$ moment with the Co moment was known.

Returning to Fig. 2, for Pt layered with the TM-dominated CoFeGd alloy, for parallel alignment of the magnetization and the beam, the dichroic spectra of both Pt and Gd are negative; therefore the $\mathrm{Pt}$ is aligned to the $\mathrm{CoFe}$ and antiparallel to the Gd. In the RE-dominated CoGd alloy, for the same parallel alignment of the net magnetization (now Gd dominated) and the field, the signs of both the Gd and Pt dichroic spectra are reversed, indicating that although the Gd moment is parallel to the magnetic field, the induced moment on the Pt is antiparallel and therefore aligned with the Co sublattice and opposite to the net magnetization. Therefore in both cases, the Pt moment aligns with the transition-metal sublattice, regardless of which sublattice dominates the magnitude and direction of the net magnetization.

These parallel and antiparallel alignments of the PIM in Pt to the net magnetization are evident in the element-specific hysteresis loops measured at the Pt and Gd edges for these alloys, see Fig. 2. Note also that the coercivity is the same as the bulk measurements in Fig. 1 and that the Pt and Gd have the same shapes and coercivities, demonstrating coupling of the Pt to the ferrimagnetic order. The alignment of the induced moment in Pt layered with RE:TM alloys may be understood by considering the exchange coupling. A quantitative description of the magnetism in RE:TM alloys is theoretically compromised by the lack of an established first-principles description of the magnetic properties of the $4 f$ system in the RE [53]; nonetheless, a two-stage mechanism of indirect exchange interactions, proposed by Campbell [54], provides a theoretical basis. In this approach, $4 f-5 d$ exchange in the $\mathrm{RE}$ induces a positive $5 d$ moment, which has subsequently been observed experimentally [55]. Direct $d-d$ interactions then mediate the magnetic coupling between the RE and TM components, the sign of which is determined by the $d$-band filling. The $3 d$ bands of $\mathrm{Co}$ and $\mathrm{Fe}$ are almost filled, which leads to ferromagnetic coupling between the TM components. Within the alloy, the $d-d$ interactions of the TM with Gd favor antiferromagnetic coupling, resulting in the ferrimagnetic structure commonly observed. Extending this model to consider the PIM of Pt in contact with a RE:TM alloy, the $5 d$ band is almost full, so coupling of the Pt moment will be ferromagnetic with $\mathrm{Co}$ and $\mathrm{Fe}$ and antiferromagnetic with $\mathrm{Gd}$, i.e., aligned parallel with the TM moments, which agrees with the experimental observations.
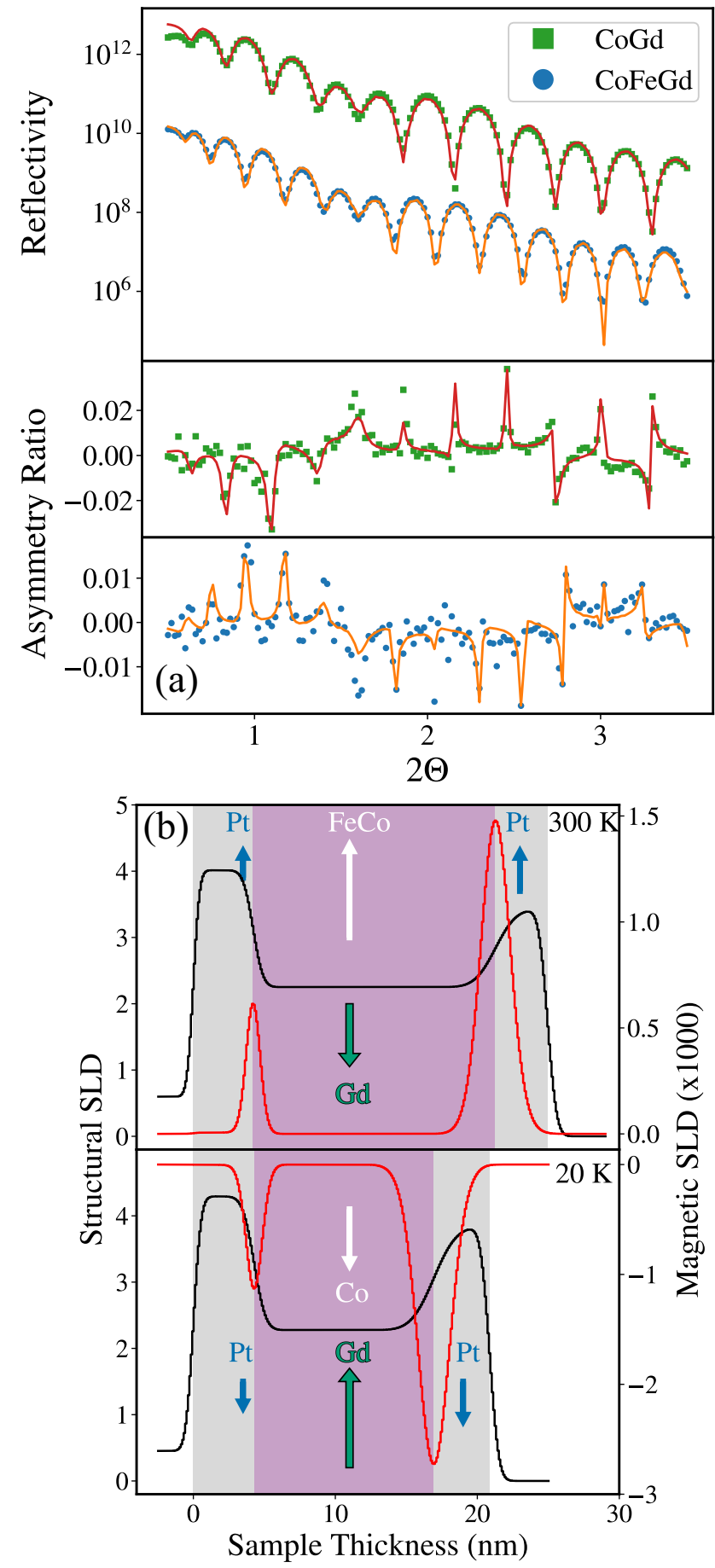

FIG. 3. Structural and magnetic characterization of the two samples using resonant $\mathrm{x}$-ray reflectivity at the $\mathrm{Pt} L_{3}$ edge. Top graph (a) shows measured reflectivity profiles with corresponding x-ray helicity asymmetry ratio below for CoGd (top) and CoFeGd (bottom). The asymmetry ratio is defined as $\left(R^{+}-R^{-}\right) /\left(R^{+}+R^{-}\right)$, where $R^{+,-}$are reflected intensities with opposite circular polarizations. The solid lines represent best-fitting models to both the structural and magnetic data. The bottom graph (b) shows the structural (black) and magnetic (red) scattering length densities from the model fits to the data for both RE:TM/Pt samples. 
Having established the presence of a Pt PIM and its ferromagnetic alignment with the transition-metal moment, analysis of the spatial relationship between the Pt PIM and the interface with the ferrimagnetic alloys provides insight into the lateral extent of the $d-d$ hybridization that gives rise to the induced moment. This requires knowledge of both the structural interface width and the PIM profile.

Examples of resonant reflectivity data at the Pt edge and the best-fitting simulations of both the specular reflectivity and the dichroic asymmetry are shown in Fig. 3. The simulations were obtained using the GenX code [56], from which the best-fitting structural (SLD) and magnetic (mSLD) scattering length densities were obtained, also shown in Fig. 3. This analysis shows that the RE:TM alloy density is uniform, indicating the $\mathrm{Gd}$ is uniformly distributed, in contrast to an observation elsewhere [29]. The SLD and mSLD indicate the structural interface width and the extent of the PIM in $\mathrm{Pt}$, respectively, while the mSLD also shows opposite signs of the induced Pt moments for the two FiMs, consistent with the earlier XMCD results. The SLD shows the interface width between the Pt and the alloy extends over $1.9 \mathrm{~nm}$ (FWHM), which is associated with a combination of topographical roughness and intermixing across the interface. The magnetic analysis shows that the interfacial profile of the PIM in Pt closely follows the structural width of the interface, supporting the notion that $d-d$ coupling is required between the Pt and the alloy components to create the PIM, which is consistent with the seminal work on layer-resolved $\mathrm{Pt}$ moments in Ni/Pt multilayers [21]. Furthermore, it is also observed that the PIM in Pt at the lower Pt/FiM interface is significantly smaller than at the upper interface, which agrees with observations in $\mathrm{Pt} / \mathrm{Co} / \mathrm{Pt}$ [3] and in $\mathrm{Pd} / \mathrm{Co} / \mathrm{Pd}$ [57], and indicates the sensitivity of the $d-d$ hybridization to the local interfacial structure, which in this case is narrower at the lower interface.

In conclusion, we have undertaken resonant $\mathrm{x}$-ray studies of proximity-induced moment (PIM) in Pt thin films layered with two rare-earth-transition-metal alloy ferrimagnets with compositions on either side of ferrimagnetic magnetization compensation at room temperature. We show the presence of a significant proximity-induced moment in the Pt layered with these ferrimagnetic alloys and that the PIM is aligned parallel to the transition-metal moments, irrespective of the direction of the net magnetization. We also find the induced moments in Pt are closely associated with the width of the interface and that the induced moment is sensitive to the details of the interfacial structure. From this we conclude that the interfacial coupling responsible for PIM in heavy metallic layers and the RE:TM alloys is intricately determined by the detailed exchange coupling between individual orbitals rather than the net moment, and that the spatial extent and amplitude of PIM are strongly tied to the details of the interfacial structure. This provides a basis for understanding the proximity-induced moment in heavy metals layered with metallic ferrimagnets in heterostructured spintronic systems.

Data presented within this article can be found at [58].

Funding is acknowledged from the EPSRC for CS 1771248, Ref. EP/P510476/1, and the Royal Society for DA, IF170030. Support is acknowledged for beam time on 4-ID-D at the Advanced Photon Source supported by the U.S. Department of Energy, Office of Science, and Office of Basic Energy Sciences under Contract No. DE-AC02-06CH11357. Travel and subsistence was funded by the EPSRC supported XMaS National Research Facility.
[1] E. Moog, J. Zak, and S. Bader, J. Appl. Phys. 69, 4559 (1991).

[2] K.-S. Ryu, L. Thomas, S.-H. Yang, and S. Parkin, Nat. Nanotechnol. 8, 527 (2013).

[3] R. Rowan-Robinson, A. Stashkevich, Y. Roussign'e, M. Belmeguenai, S. Ch'erif, A. Thiaville, T. Hase, A. Hindmarch, and D. Atkinson, Sci. Rep. 7, 16835 (2017).

[4] S. Rezende, R. Rodríguez-Suárez, M. Soares, L. Vilela-Leão, D. Ley Domínguez, and A. Azevedo, Appl. Phys. Lett. 102, 012402 (2013).

[5] S. Dushenko, M. Hokazono, K. Nakamura, Y. Ando, T. Shinjo, and M. Shiraishi, Nat. Commun 9, 3118 (2018).

[6] M.-H. Nguyen, C.-F. Pai, K. X. Nguyen, D. A. Muller, D. C. Ralph, and R. A. Buhrman, Appl. Phys. Lett. 106, 222402 (2015).

[7] W. Zhang, W. Han, X. Jiang, S.-H. Yang, and S. S. Parkin, Nat. Phys. 11, 496 (2015).

[8] C.-F. Pai, Y. Ou, L. H. Vilela-Leão, D. C. Ralph, and R. A. Buhrman, Phys. Rev. B 92, 064426 (2015).

[9] M. Tokac, S. A. Bunyaev, G. N. Kakazei, D. S. Schmool, D. Atkinson, and A. T. Hindmarch, Phys. Rev. Lett. 115, 056601 (2015).

[10] S. Azzawi, A. Ganguly, M. Tokac, R. M. Rowan-Robinson, J. Sinha, A. T. Hindmarch, A. Barman, and D. Atkinson, Phys. Rev. B 93, 054402 (2016).
[11] C. Swindells, A. T. Hindmarch, A. J. Gallant, and D. Atkinson, Phys. Rev. B 99, 064406 (2019).

[12] M. Caminale, A. Ghosh, S. Auffret, U. Ebels, K. Ollefs, F. Wilhelm, A. Rogalev, and W. E. Bailey, Phys. Rev. B 94, 014414 (2016).

[13] L. J. Zhu, D. C. Ralph, and R. A. Buhrman, Phys. Rev. B 98, 134406 (2018).

[14] S. Y. Huang, X. Fan, D. Qu, Y. P. Chen, W. G. Wang, J. Wu, T. Y. Chen, J. Q. Xiao, and C. L. Chien, Phys. Rev. Lett. 109, 107204 (2012).

[15] S. Geprägs, S. Meyer, S. Altmannshofer, M. Opel, F. Wilhelm, A. Rogalev, R. Gross, and S. T. Goennenwein, Appl. Phys. Lett. 101, 262407 (2012).

[16] S. Shimizu, K. S. Takahashi, T. Hatano, M. Kawasaki, Y. Tokura, and Y. Iwasa, Phys. Rev. Lett. 111, 216803 (2013).

[17] H. Nakayama, M. Althammer, Y.-T. Chen, K. Uchida, Y. Kajiwara, D. Kikuchi, T. Ohtani, S. Geprägs, M. Opel, S. Takahashi et al., Phys. Rev. Lett. 110, 206601 (2013).

[18] H. Wu et al., IEEE Trans. Magn. 51, 4100104 (2015).

[19] W. Amamou, I. V. Pinchuk, A. H. Trout, R. E. A. Williams, N. Antolin, A. Goad, D. J. O'Hara, A. S. Ahmed, W. Windl, D. W. McComb, and R. K. Kawakami, Phys. Rev. Mater. 2, 011401 (2018). 
[20] J. Vogel, A. Fontaine, V. Cros, F. Petroff, J.-P. Kappler, G. Krill, A. Rogalev, and J. Goulon, Phys. Rev. B 55, 3663 (1997).

[21] F. Wilhelm, P. Poulopoulos, G. Ceballos, H. Wende, K. Baberschke, P. Srivastava, D. Benea, H. Ebert, M. Angelakeris, N. K. Flevaris, D. Niarchos, A. Rogalev, and N. B. Brookes, Phys. Rev. Lett. 85, 413 (2000).

[22] F. Wilhelm, P. Poulopoulos, H. Wende, A. Scherz, K. Baberschke, M. Angelakeris, N. K. Flevaris, and A. Rogalev, Phys. Rev. Lett. 87, 207202 (2001).

[23] F. Wilhelm, M. Angelakeris, N. Jaouen, P. Poulopoulos, E. T. Papaioannou, C. Mueller, P. Fumagalli, A. Rogalev, and N. K. Flevaris, Phys. Rev. B 69, 220404(R) (2004).

[24] M. Collet, R. Mattana, J.-B. Moussy, K. Ollefs, S. Collin, C. Deranlot, A. Anane, V. Cros, F. Petroff, F. Wilhelm et al., Appl. Phys. Lett. 111, 202401 (2017).

[25] M. Valvidares, N. Dix, M. Isasa, K. Ollefs, F. Wilhelm, A. Rogalev, F. Sánchez, E. Pellegrin, A. Bedoya-Pinto, P. Gargiani et al., Phys. Rev. B 93, 214415 (2016).

[26] Y. M. Lu, Y. Choi, C. M. Ortega, X. M. Cheng, J. W. Cai, S. Y. Huang, L. Sun, and C. L. Chien, Phys. Rev. Lett. 110, 147207 (2013).

[27] S. Nodo, S. Ono, T. Yanase, T. Shimada, and T. Nagahama, Appl. Phys. Express 13, 063004 (2020).

[28] Ł. Fraçkowiak, P. Kuświk, M. Urbaniak, G. D. ChavesO’Flynn, and F. Stobiecki, Sci. Rep. 8, 16911 (2018).

[29] N. Bergeard, A. Mougin, M. Izquierdo, E. Fonda, and F. Sirotti, Phys. Rev. B 96, 064418 (2017).

[30] M. Binder, A. Weber, O. Mosendz, G. Woltersdorf, M. Izquierdo, I. Neudecker, J. R. Dahn, T. D. Hatchard, J.-U. Thiele, C. H. Back, and M. R. Scheinfein, Phys. Rev. B 74, 134404 (2006).

[31] Y. Hirata, D. H. Kim, T. Okuno, T. Nishimura, D. Y. Kim, Y. Futakawa, H. Yoshikawa, A. Tsukamoto, K. J. Kim, S. B. Choe, and T. Ono, Phys. Rev. B 97, 220403(R) (2018).

[32] K. J. Kim, S. K. Kim, Y. Hirata, S. H. Oh, T. Tono, D. H. Kim, T. Okuno, W. S. Ham, S. Kim, G. Go, Y. Tserkovnyak, A. Tsukamoto, T. Moriyama, K. J. Lee, and T. Ono, Nat. Mater. 16, 1187 (2017)

[33] S. A. Siddiqui, J. Han, J. T. Finley, C. A. Ross, and L. Liu, Phys. Rev. Lett. 121, 057701 (2018).

[34] R. F. Evans, T. A. Ostler, R. W. Chantrell, I. Radu, and T. Rasing, Appl. Phys. Lett. 104, 082410 (2014).

[35] T. A. Ostler, J. Barker, R. F. Evans, R. W. Chantrell, U. Atxitia, O. Chubykalo-Fesenko, S. El Moussaoui, L. Le Guyader, E. Mengotti, L. J. Heyderman, F. Nolting, A. Tsukamoto, A. Itoh, D. Afanasiev, B. A. Ivanov, A. M. Kalashnikova, K. Vahaplar, J. Mentink, A. Kirilyuk, T. Rasing, and A. V. Kimel, Nat. Commun. 3, 666 (2012).

[36] Y. Hirata, D. H. Kim, S. K. Kim, D. K. Lee, S. H. Oh, D. Y. Kim, T. Nishimura, T. Okuno, Y. Futakawa, H. Yoshikawa,
A. Tsukamoto, Y. Tserkovnyak, Y. Shiota, T. Moriyama, S. B. Choe, K. J. Lee, and T. Ono, Nat. Nanotechnol. 14, 232 (2019).

[37] S. K. Kim, K. J. Lee, and Y. Tserkovnyak, Phys. Rev. B 95, 140404(R) (2017).

[38] J. Finley and L. Liu, Phys. Rev. Appl. 6, 054001 (2016).

[39] R. Mishra, J. Yu, X. Qiu, M. Motapothula, T. Venkatesan, and H. Yang, Phys. Rev. Lett. 118, 167201 (2017).

[40] N. Roschewsky, T. Matsumura, S. Cheema, F. Hellman, T. Kato, S. Iwata, and S. Salahuddin, Appl. Phys. Lett. 109, 112403 (2016).

[41] N. Roschewsky, C. H. Lambert, and S. Salahuddin, Phys. Rev. B 96, 064406 (2017).

[42] J. Han, A. Richardella, S. A. Siddiqui, J. Finley, N. Samarth, and L. Liu, Phys. Rev. Lett. 119, 077702 (2017).

[43] O. Inyang, A. Rafiq, C. Swindells, S. Ali, and D. Atkinson, Sci. Rep. 10, 1 (2020).

[44] L. Frackowiak, P. Kuświk, G. D. Chaves-O’Flynn, M. Urbaniak, M. Matczak, P. P. Michalowski, A. Maziewski, M. Reginka, A. Ehresmann, and F. Stobiecki, Phys. Rev. Lett. 124, 047203 (2020).

[45] T. Kimura, Y. Otani, T. Sato, S. Takahashi, and S. Maekawa, Phys. Rev. Lett. 98, 156601 (2007).

[46] R. Ramaswamy, J. M. Lee, K. Cai, and H. Yang, Appl. Phys. Rev. 5, 031107 (2018).

[47] J. P. Andrés, J. A. González, T. P. A. Hase, B. K. Tanner, and J. M. Riveiro, Phys. Rev. B 77, 144407 (2008).

[48] F. Leuenberger, A. Parge, W. Felsch, F. Baudelet, C. Giorgetti, E. Dartyge, and F. Wilhelm, Phys. Rev. B 73, 214430 (2006).

[49] G. Champion, N. Lalioti, V. Tangoulis, M. A. Arrio, P. Sainctavit, F. Villain, A. Caneschi, D. Gatteschi, C. Giorgetti, F. Baudelet, M. Verdaguer, and C. C. Dit Moulin, J. Am. Chem. Soc. 125, 8371 (2003).

[50] Y. C. Tseng, D. Haskel, J. C. Lang, Y. Mudryk, V. K. Pecharsky, and K. A. Gschneidner, J. Appl. Phys. 103, 07B301 (2008).

[51] R. Streubel, C. H. Lambert, N. Kent, P. Ercius, A. T. N'Diaye, C. Ophus, S. Salahuddin, and P. Fischer, Adv. Mater. 30, 1800199 (2018).

[52] Y. C. Lau, Z. Chi, T. Taniguchi, M. Kawaguchi, G. Shibata, N. Kawamura, M. Suzuki, S. Fukami, A. Fujimori, H. Ohno, and M. Hayashi, Phys. Rev. Mater. 3, 104419 (2019).

[53] T. Miyake and H. Akai, J. Phys. Soc. Jpn. 87, 041009 (2018).

[54] I. A. Campbell, J. Phys. F Met. Phys. 2, L47 (1972).

[55] O. S. Anilturk and A. R. Koymen, Phys. Rev. B 68, 024430 (2003).

[56] M. Björck and G. Andersson, J. Appl. Crystallogr. 40, 1174 (2007).

[57] D.-O. Kim, K. M. Song, Y. Choi, B.-C. Min, J.-S. Kim, J. W. Choi, and D. R. Lee, Sci. Rep. 6, 1 (2016).

[58] C. Swindells, Proximity induced magnetism in Pt layered with rare-earth:transition metal ferrimagnetic alloys, http://doi.org/ 10.15128/r2765371377 (2020). 